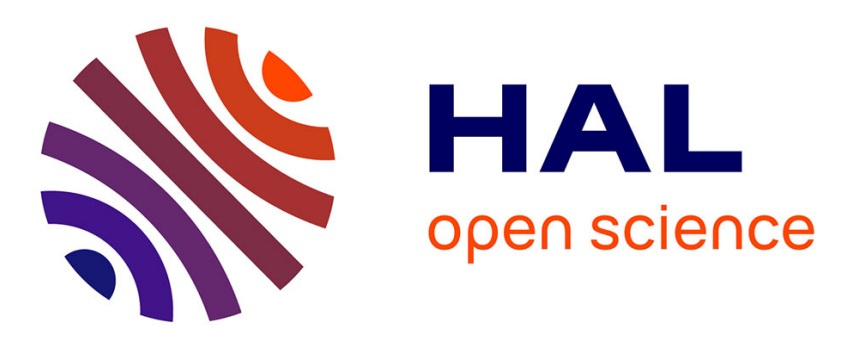

\title{
Supporting Efficient and Advanced Omniscient Debugging for xDSMLs
}

Erwan Bousse, Jonathan Corley, Benoit Combemale, Jeff Gray, Benoit Baudry

\section{To cite this version:}

Erwan Bousse, Jonathan Corley, Benoit Combemale, Jeff Gray, Benoit Baudry. Supporting Efficient and Advanced Omniscient Debugging for xDSMLs. 8th International Conference on Software Language Engineering (SLE), Oct 2015, Pittsburg, United States. hal-01182517v2

\section{HAL Id: hal-01182517 \\ https://hal.inria.fr/hal-01182517v2}

Submitted on 21 Aug 2015

HAL is a multi-disciplinary open access archive for the deposit and dissemination of scientific research documents, whether they are published or not. The documents may come from teaching and research institutions in France or abroad, or from public or private research centers.
L'archive ouverte pluridisciplinaire HAL, est destinée au dépôt et à la diffusion de documents scientifiques de niveau recherche, publiés ou non, émanant des établissements d'enseignement et de recherche français ou étrangers, des laboratoires publics ou privés. 


\section{Supporting Efficient and Advanced Omniscient Debugging for xDSMLs}

\author{
Erwan Bousse \\ Irisa \\ Rennes, France \\ erwan.bousse@irisa.fr
}

\author{
Jonathan Corley \\ University of Alabama \\ Tuscaloosa, AL, USA \\ corle001@crimson.ua.edu
}

\author{
Benoit Combemale \\ Inria \\ Rennes, France \\ benoit.combemale@inria.fr
}

\author{
Jeff Gray \\ University of Alabama \\ Tuscaloosa, AL, USA \\ gray@cs.ua.edu
}

\author{
Benoit Baudry \\ Inria \\ Rennes, France \\ benoit.baudry@inria.fr
}

\begin{abstract}
Omniscient debugging is a promising technique that relies on execution traces to enable free traversal of the states reached by a system during an execution. While some GeneralPurpose Languages (GPLs) already have support for omniscient debugging, developing such a complex tool for any executable Domain-Specific Modeling Language (xDSML) remains a challenging and error prone task. A solution to this problem is to define a generic omniscient debugger for all xDSMLs. However, generically supporting any xDSML both compromises the efficiency and the usability of such an approach. Our contribution relies on a partly generic omniscient debugger supported by generated domain-specific trace management facilities. Being domain-specific, these facilities are tuned to the considered XDSML for better efficiency. Usability is strengthened by providing multidimensional omniscient debugging. Results show that our approach is on average 3.0 times more efficient in memory and 5.03 more efficient in time when compared to a generic solution that copies the model at each step.
\end{abstract}

\section{Categories and Subject Descriptors $\quad$ D.2.5 [Software Engi-} neering]: Testing and Debugging

Keywords Omniscient Debugger, xDSML, Execution Trace

\section{Introduction}

Many recent efforts aim at providing facilities to design executable Domain-Specific Modeling Languages (xDSMLs) 4 13, 17]. xDSMLs allow system engineers to analyze behavioral properties early in the development process. In particular, debugging is a common dynamic facility to observe and control an execution in order to better understand a behavior or to look for the cause of a defect. However, standard debugging only provides facilities to pause and step forward during an execution, hence requiring developers to restart from the beginning to give a second look at a state of interest. To cope with this issue, omniscient debugging is a promising technique that relies on execution traces to enable free traversal of the states reached by a system, thereby allowing developers to "go back in time." [10]

While most general-purpose languages (GPLs) already have their own efficient standard debugger (e.g., Java ${ }^{1}$ ) or omniscient debugger (e.g., also Java [16]), developing such a complex tool for any XDSML remains a difficult and error prone task. Despite the specificities of each XDSML, it is possible to identify a common set of debugging facilities for all xDSMLs. Thus, to avoid manual creation of each debugger, a possible solution is to define a generic omniscient debugger that would work for any xDSML. However, handling any xDSML has two main consequences: (1) There is necessarily a trade-off between genericity and efficiency of the debugging operations, since supporting any XDSML requires the use of expensive introspection, conditionals, or type checks to support a wide variety of abstract syntax and runtime data structures. Moreover, since debugging is an interactive activity, responsiveness is of primary importance. Hence, a first concern is the efficiency of a generic debugger. (2)

\footnotetext{
1 http://docs.oracle.com/javase/8/docs/technotes/tools/unix/jdb.html
} 
The execution data structure defined in an xDSML can be arbitrarily complex (e.g., a large object-oriented structure), and therefore difficult to comprehend in a debugging session, especially if the execution leads to a large amount of states. Hence, a second concern is the usability of omniscient debugging for XDSMLs; i.e., specific advanced facilities are required to manage the complexity and size of the executions. To summarize, the following are key objectives that drive the focus of this paper:

O\#1: Providing efficient omniscient debugging facilities, to ensure responsiveness of the debugger.

O\#2: Offering advanced omniscient debugging facilities, to improve the usability.

To address $\mathrm{O \# 1}$, we propose to go from a generic omniscient debugger to a generic meta-approach to define omniscient debuggers. Such a generative approach can provide an efficient and finely tuned omniscient debugger for any xDSML. Yet, considering a generic set of debugging services for all xDSMLs, both the interface and some underlying logic of a debugger can remain generic without compromising efficiency. Hence, our contribution relies on a partially generic omniscient debugger supported by generated domainspecific trace management facilities. The trace management facilities include a domain-specific trace metamodel that precisely captures the execution state of a model conforming to the $\mathrm{xDSML}$, and a domain-specific trace manager providing all the required services to manipulate the execution trace generically. Because the trace manager is domain-specific, it is finely tuned to the considered XDSML and to the generated trace metamodel, and hence more efficient than a generic one. Previously, we have separately explored both supporting efficient, generic omniscient debugging services for model transformations [6] and generating domain-specific trace metamodels [2]. In this paper, we explore adapting domain-specific trace metamodels to support generic omniscient debugging services for xDSMLs. To address $\mathrm{O \# 2}$. our contribution provides multidimensional omniscient debugging services, which mix both omniscient debugging services, and advanced facilities to navigate among the values of specific elements of the executed model.

We implemented our approach as part of the GEMOC Studio, a language and modeling workbench; and we conducted an empirical evaluation. To evaluate the efficiency of our solution, we assessed its quality with regard to both memory consumption and the time required to run omniscient debugging operations. We compared our approach with two generic omniscient debuggers: one that simulates omniscient debugging by resetting the execution engine and re-executing until the target state is reached, and one that copies the model at each execution step. Obtained results show that our approach is on average 3.0 times more efficient in memory when compared to the second debugger, and respectively 54.1 and 5.03

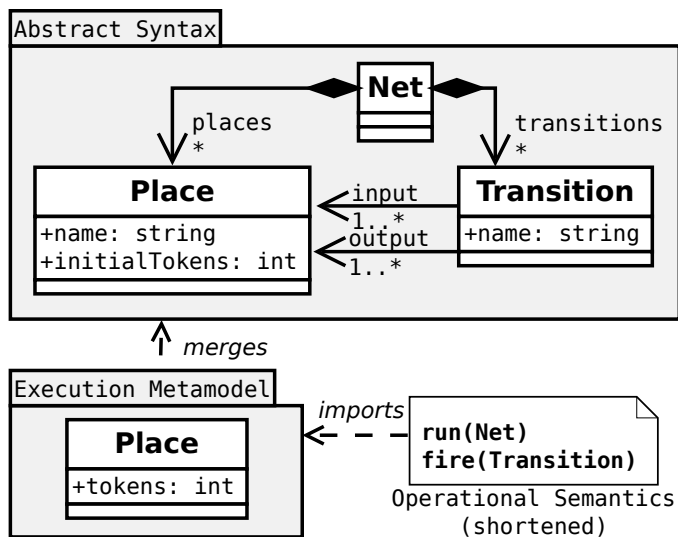

Figure 1. Petri Net xDSML

times more efficient in time when compared respectively to the first and the second debugger.

The remaining sections are as follows. Section 2 defines the considered scope of model execution and model debugging. Section 3 presents our generative approach to provide generic multidimensional omniscient debugging. Section 4 describes a prototype supporting the technique in the GEMOC Studio. Section 5 discusses the evaluation of our approach. Finally, Section 6 discusses related work and Section 7 concludes the paper.

\section{Model Execution and Model Debugging}

In this section, we precisely define the scope of the considered executable models, their specification into xDSMLs, and the resulting execution trace corresponding to the application of the latter to the former. Then we define the scope of the advanced omniscient debugging facilities proposed to analyze executable models.

\subsection{Model Execution}

$\boldsymbol{x D S M L}$ We consider a metamodel to be an object-oriented model defining a particular domain. Thus, it is composed of classes that consist of properties. A property is either an attribute (typed by a primitive type) or a reference to another class. A model is then a set of objects that conforms to a metamodel. Conformity implies each object in the model is an instance of one class defined in the metamodel. An object is composed of fields, each representing the object's values for one property of the corresponding class.

While the purpose of metamodeling is to define languages, executable metamodeling also includes defining execution semantics within the language definition. This is done through $\mathrm{xDSMLs}$, languages that include the definitions of the execution state of a model conforming to the language, and execution semantics that operate on this state.

There are two different approaches to define execution semantics: translational semantics and operational semantics. We focus in this paper on the case of operational semantics, and we plan to consider translational semantics in the future. 


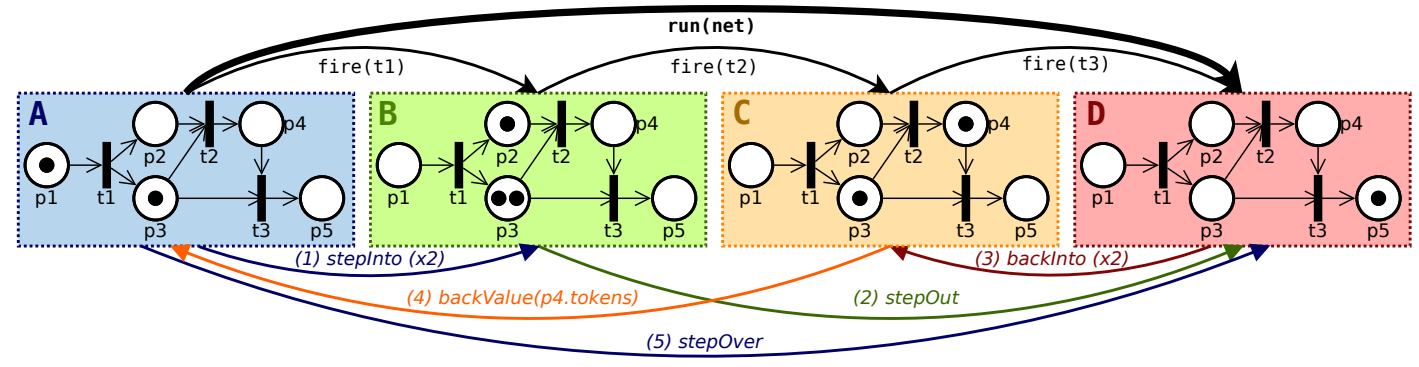

Figure 2. Example of Petri Net execution trace annotated with the use of a selection of debugging services

\section{Definition 1 An xDSML is defined by:}

- An abstract syntax, that is a metamodel. We call immutable property a property introduced in this metamodel. At the model level, we call immutable field an object's field based on an immutable property.

- An execution metamodel, that extends the abstract syntax by package merge ${ }^{2}$ We call a property introduced in this metamodel a mutable property. In a model, a mutable field is an object's field based on a mutable property.

- Operational semantics, that are a set of transformation rules that modify a model conforming to the execution metamodel by changing values of mutable fields and creating/destroying instances of classes introduced in the execution metamodel.

- An initialization function that, when given a model conforming to the abstract syntax, returns a model conforming to the execution metamodel.

Figure 1 shows an example of a Petri Net xDSML. At the top, its abstract syntax is depicted with three classes Net, Place and Transition. At the bottom-left is the execution metamodel, that extends the class Place using package merge with a new mutable property tokens. The initialization function (not shown) transforms each original object into an executable object (e.g., a Place object gains a tokens field) as defined in the execution metamodel. The function also initializes each tokens field with the value of initialTokens. At the bottom-right are the signatures of the rules defined in the operational semantics. The rules are defined as follows: run continuously looks for enabled transitions, and uses fire to transfer tokens from input to output places of such transitions. Note that a Place object cannot be created during execution, since the Place class was introduced in the abstract syntax.

Model Execution Given an xDSML, executing a conforming model consists of the application, on demand, of the transformation rules that define its operational semantics.

Execution Trace Execution traces aim at capturing both the changes in the mutable fields of the model, and the applied transformation rules that led to these changes. While

\footnotetext{
${ }^{2}$ Note that in practice, existing tools and approaches use different but similar extension mechanisms; e.g., Kermeta [9] uses aspect weaving and xMOF [13] uses generalization.
}

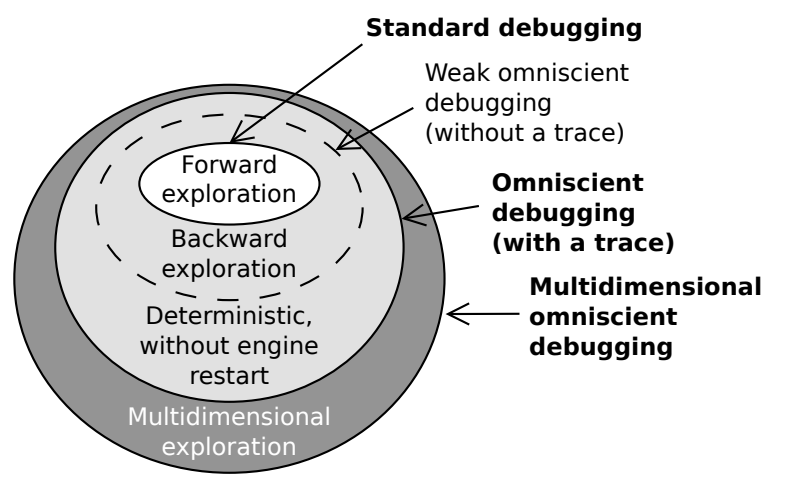

Figure 3. Feature comparison of the debugging approaches

traces can take various forms, this work considers that an execution trace is a sequence of states capturing the values of the mutable fields and steps capturing the application of transformation rules.

Definition 2 An execution trace is defined by:

- A sequence of execution states, each capturing the values of all mutable fields in the model.

- A set of steps, each being the application of a transformation rule of the operational semantics. A step going from a state to its following state is called a small step, while a step containing several steps is called a big step. In other words, a big step is the application of a transformation rule that relies on other existing rules.

Figure 2 presents a trace from the execution of a Petri Net model conforming to the Petri Net XDSML shown in Figure 1 The trace is composed of four states, on top of which the steps of the execution are depicted. States are separated by three small steps that represent the applications of the fire transformation rule. A big step goes from the first state to the last state to represent the application of the run rule. Note that the bottom part of the figure (i.e., the colored arrows) is described later in Section 2.2.

\subsection{Model Debugging}

Debugging Approaches Debugging an executable model involves controlling the model's execution and observing the states traversed. Figure 3 shows four approaches to achieve this, with different levels of control over the execution. First, 
standard debugging only traverses forward through the states reached by the model through the application of the operational semantics rules. Second, we call weak omniscient debugging the possibility to go backward in the exploration of the states through a restart of the execution engine and a re-execution until the target state is reached. Note that this can be accomplished manually with any standard debugger. Third, omniscient debugging relies on an execution trace to revert the executed model into a prior state. Using a trace makes the procedure deterministic (i.e., the exact same states are visited) even if the model or the operational semantics are non-deterministic. Finally, our proposal relies on multidimensional omniscient debugging, which adds facilities to navigate among the values of mutable fields of the model. In the remainder of this section, we present these debugging approaches as sets of provided services. Note that all these services are only valid when the execution is paused; i.e., when the execution engine waits for instruction before applying a transformation rule.

Standard Debugging Most debuggers only provide standard debugging, which includes the following forward exploration services:

- breakpoint: pause the execution when a specified condition is true (e.g., a transformation rule is reached).

- steplnto: resume execution and pauses after either executing a single small step or moving to the next step encountered in the following big step.

- stepOver: resume execution and pause when the next step is completed (including the contained steps, if this is a big step).

- stepOut: resume execution and pause when the first step not contained within the current big step is reached.

- play: resume execution.

- visualization of the current state: display the values of relevant mutable elements.

Omniscient Debugging To provide exploration of previously visited states, omniscient debugging relies on the construction of an execution trace to extend standard debugging with the following services:

- jump: revert the model to a specified state.

- backInto: revert a single small step or moves to the last step encountered in a big step.

- backOver: revert the last encountered step (including the contained steps, if the last step is a big step).

- backOut: revert all the remaining steps within the current big step.

- playBackwards: continuously revert execution until the execution is paused or the initial state is reached.
- visualization of the trace: display an interactive representation of the reached execution states and show which state is current.

Multidimensional Omniscient Debugging With the ability to go both forward and backward, a developer can explore any state of a model's execution. Yet, large traces are difficult to navigate practically, and information stored within a state can be arbitrarily complex, compromising usability (O\#2. To cope with this issue, we investigate multidimensional omniscient debugging; i.e., facilities to navigate among the values of the mutable fields of the model:

- jumpValue: jump to the first state in which a given mutable field has a given value.

- step Value: given a mutable field, jump to the next value of this field.

- backValue: given a mutable field, jump to the previous value of this field.

- visualization of the value sequences: display an interactive representation of the reached values of the mutable fields and show which values are the current ones.

Example Debugging Scenario Consider a complete execution and debugging scenario with a Petri Net model conforming to the xDSML shown in Figure 1. The initial state of the considered Petri Net model is depicted at the left of Figure 2 with the label A. First, we set a breakpoint in order to pause the execution right after it starts. Then we start the execution and reach the first $A$ state. From there, the next step is an application of run. We perform a first steplnto (1), which does not change the current state, but presents us with a new next step, which is an application of fire on $t 1$. We then use steplnto a second time (1), which applies the fire small step and brings us to the B state. From there, we use stepOut (2) to get out from the current big step (i.e., run ), which brings us to the D state. At this point, the trace is fully constructed, and no additional transformation rules will be applied.

Then, similar to the beginning of the scenario, we apply twice backInto (3) to reach the $C$ state. We then use backValue (4) to go back to the previous value reached by the tokens field of the p4 Place object. While p4 has one token in the $C$ state, its previous amount was zero, which started in the A state. Hence, we reach the A state again. Finally, this time we use stepOver (5) to directly follow the first step (i.e., run) and we reach the $\mathrm{D}$ state again. Note that in this case, stepOver should not apply any transformation rules, but simply read information from the execution trace to directly revert the executed model into the stored $\mathrm{D}$ state.

\section{Efficient and Advanced Omniscient Debugging for xDSMLs}

This section presents our approach that provides efficient and advanced omniscient debugging for xDSMLs using a partially 


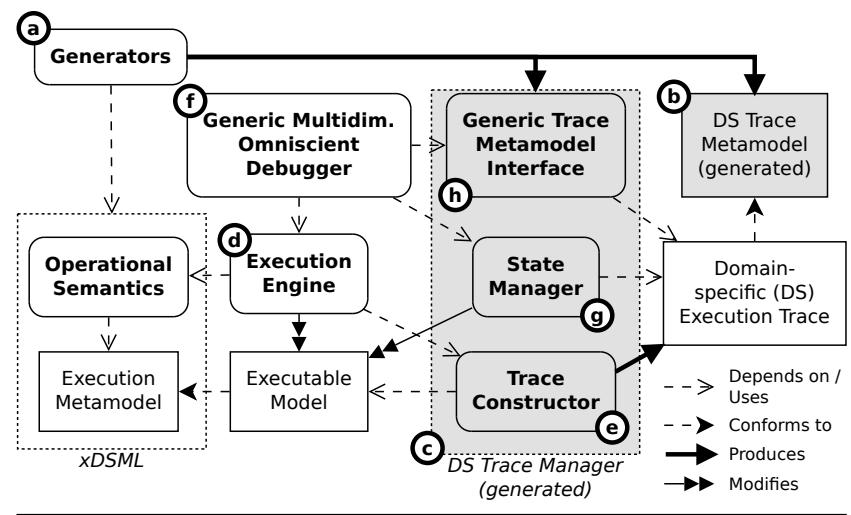

Figure 4. Overview of the approach

generic, multidimensional omniscient debugger supported by generated domain-specific trace management facilities.

\subsection{Overview of the Approach}

Defining an xDSML implies the definition of a number of domain-specific facilities to edit or analyze a model conforming to the language. In particular, one method to provide a visual animation of a model execution is to observe the model and react to changes. Because such a pattern is common when defining tools for xDSMLs, our approach is designed to have a single instance of the executed model loaded at any given time that can be modified throughout the execution and the debugging session.

Figure 4 shows an overview of our approach. We consider that the initialization function of the xDSML was already applied to an input model, creating the executable model. The first step of our approach relies on generators (a), which take the considered XDSML as input to produce two domainspecific components: a trace metamodel (b) and a trace manager (c). The second step is the execution and the debugging of the model. The execution engine (d) applies the operational semantics to change the model and uses the trace constructor (e) from the trace manager to construct a domain-specific trace. The generic multidimensional omniscient debugger $(\mathrm{f})$ provides all the services described in Section 2 by controlling the execution engine and relying on the state manager $(\mathrm{g})$ to revert the model into previous states. Additionally, the debugger relies on the generic trace metamodel interface $(\mathrm{h})$ to manipulate the trace.

To illustrate a subset of the interactions between the components shown in Figure 4, Figure 5 shows a sequence diagram that sketches what happens when a small step must be computed and stored in the trace. Duration bars depicted in gray represent changes made in the affected element. First, the engine (d) determines the next rule to apply then notifies the trace constructor (e) that a small step will occur. As a result, the trace constructor reads the executed model, and updates the domain-specific trace with new elements accordingly (e.g., add a new small step and, if the model was

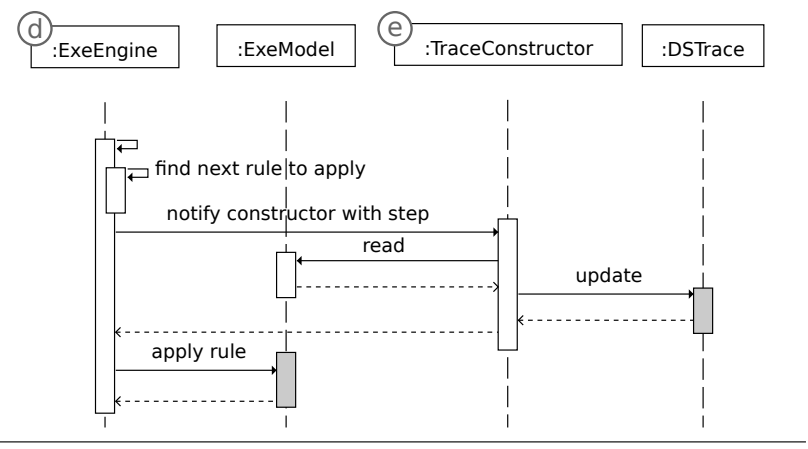

Figure 5. Interactions when a small step is to be computed and added to the trace

altered, a new state). Finally, the execution engine applies the rule and modifies the executed model accordingly.

We present all of these components in more detail in the remainder of this section.

\subsection{Execution Engine}

First and foremost, an omniscient debugger must provide precise control over the execution of a model, such as the ability to pause during execution or traverse the trace in a controlled manner. For this to be possible, the execution engine (d) must adhere to certain specifications. The engine must be able to drive the execution of the model (i.e., initialization, start, stop), and to provide to the debugger some control over the execution. This includes the ability to pause the execution at a specific state during execution, and the ability to resume the execution from a paused state. We assume that the engine provides at least the following services:

- pauseWhen: order to suspend the execution in between two transformation rule applications as soon as a given predicate is true.

- isPaused: return true if the engine is paused.

- resume: resume execution (i.e., cancel a pause).

As presented in Section 4.1, we developed an execution engine that encompasses the aforementioned services.

\subsection{Domain-Specific Trace Metamodel}

In our prior work [2], we presented a generative approach that automatically provides a rich domain-specific trace metamodel for an XDSML. Instead of relying on clones of the executed model to construct a trace, the metamodel precisely captures its execution state through an efficient object-oriented structure based on the mutable properties of the XDSML. In addition, the structure provides rich navigation facilities to browse a trace according to the values reached by the mutable fields of the model. To benefit from such efficient trace structures (O\#1), we rely on this approach for the automatic generation (a in Figure 4) of the domain-specific trace metamodel (b in Figure 4). 


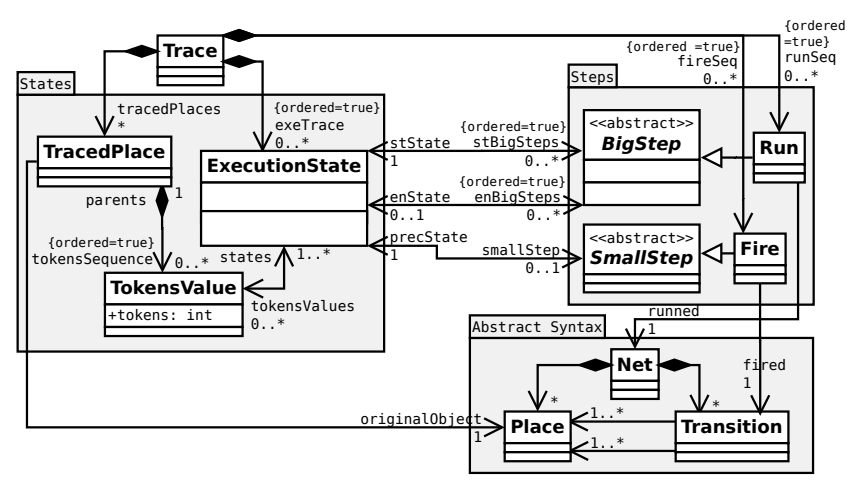

Figure 6. Petri net rich domain-specific trace metamodel

To support omniscient debugging, we extended this generative approach with the notion of big step, in addition to small step. This included adding a BigStep class to the base classes that are generated, the derivation of domain-specific big step classes from the XDSML operation definitions, and extending the generation algorithm to create inheritance links from big step classes to BigStep.

Figure 6 shows an example of a rich domain-specific trace metamodel for Petri Nets using our approach. As an overview, the state of the model is captured in the ExecutionState class, which is composed of a tuple of TokensValue objects, with each representing the value of a tokens field at a given point in time. Each TracedPlace object captures all the values of the mutable fields of a specific Place object of the executed model. On the right, steps are represented by Run and Fire, which inherit BigStep and SmallStep respectively.

\subsection{Trace Constructor}

To provide omniscient debugging, we must construct an execution trace during the execution of the model. We have defined the following set of operations to be provided by the trace constructor (e in Figure 4):

- initialize: create the base elements of the trace.

- addState: add a new state in the trace if a mutable field of the model changed, or if instances of classes introduced in the execution metamodel are created/deleted.

- addSmallStep: add a small step in the trace.

- bigStepStarted: notify that a big step has started.

- bigStepEnded: notify that a big step has ended.

As explained in Section 2.1, the execution of a model consists of the application of a sequence of transformation rules. To capture an execution state that matches a model conforming to the execution metamodel, the operation addState must be called just before or after the transformation rule.

Since a big step is simply a sequence of small steps, we only need to capture states before and after small steps. However, we also need to capture when steps occur, hence addSmallStep must be called at each transformation rule that matches a small step, while bigStepStarted and bigSte$p$ Ended must be called before and after a rule matching a big step, respectively. In summary, all the calls required to construct the trace are as follows:

- Just before the first small step: initialization

- Just before a small step: addState, addSmallStep

- After the last small step: addState

- Just before a big step: bigStepStarted

- Just after a big step: bigStepEnded

\subsection{Generic Trace Metamodel}

Our approach relies on the generation of a domain-specific trace metamodel for the considered xDSML. Since the debugger is generic, an interface must also be defined to manipulate traces in a generic way despite their various possible data structures. We defined this structural interface as a generic trace metamodel ( $\mathrm{h}$ in Figure 4) specifying all the information that should be accessible within a domain-specific trace. Thus, it has a similar structure to generated domain-specific trace metamodels, except it contains less classes and properties.

Figure 7 shows the generic trace metamodel interface. To summarize, we have the same base classes (Trace and ExecutionState) as generated domains specific trace metamodels (e.g., the Petri net trace metamodel shown in Figure 6), and classes to represent both steps (ExecutionStep) and values (TracedObject, ValueSequence, Value). Primitive types that extend the Value class (e.g., IntegerValue) are not shown due to space limitations. We use references to elements of the execution metamodel, operational semantics, and executed model: appliedRule to specify which rule was applied, originalObject to specify which object of the original model is traced by a TracedObject, and tracedProperty to specify the property traced by a ValueSequence. Also note that derived properties are defined to facilitate the navigation among the trace, such as nextState. Finally, ExecutionStep objects are ordered either by starting time, or by ending time, hence the derived properties nextStarting and previousStarting for the starting time then nextEnding and previousEnding for the ending time.

In order to go back and forth through the execution states and steps, a Trace has a reference currentStepForward to the ExecutionStep object that represents the next forward execution step, and a similar reference currentStepBackward for the next backward step (e.g., to backOver the last step handled by the debugger). The current state is accessible with currentState, which is derived from currentStepForward. Similarly, the property currentValue of ValueSequence is indirectly derived from currentState.

To provide this interface, our solution relies on the generation of a one-way model transformation from the domain specific trace metamodel to the generic trace metamodel. Thereby, we have a generic read-access to the trace. Regarding write-accesses, we store the debugging state 


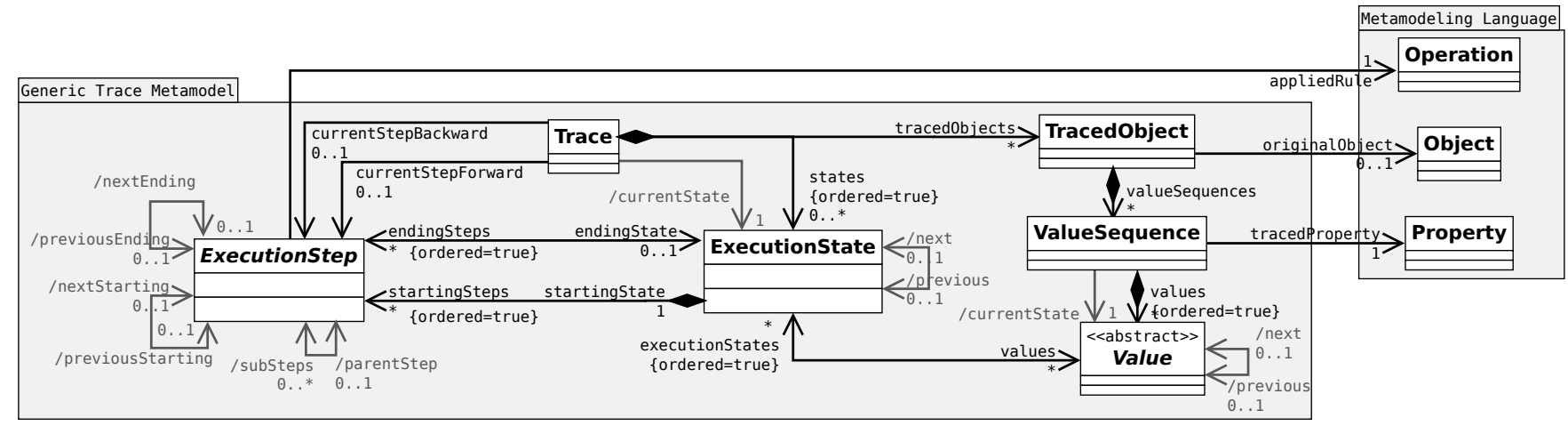

Figure 7. Generic Trace Metamodel Interface

(e.g., currentState) in a separate generic structure, hence avoiding the need to modify the domain-specific trace.

\subsection{State Manager}

An omniscient debugger must be able to revisit a previous state by reverting the executed model into the state stored in the execution trace. The operation enabling a debugger to return to a past state is provided by the state manager ( $\mathrm{g}$ in Figure 4p, which we specified with a single service:

- restoreModelToState: restore the executed model into a given execution state.

The idea is similar to the well-studied memento design pattern, albeit at the model level. The originator is the model being executed; the memento is an execution state of the trace; and the caretaker is both the trace and the trace manager.

\subsection{Domain-Specific Trace Manager}

To implement both the trace constructor and the state manager and to generically expose as much information as stated in the generic trace metamodel, our approach relies on the generation of a domain-specific trace manager ( $c$ in Figure 47. The reason for generating this component is efficiency (O\#1, because trace manipulations can be tuned for both the considered XDSML and the generated domainspecific trace metamodel (introduced in Section 3.3.

Consequently, the domain-specific trace manager generation is coupled with the domain-specific trace metamodel generation. Since all generated operations manipulate a trace conforming to this metamodel, a set of traceability links obtained from the generation of the domain-specific trace metamodel is provided to the generator. From there, the main steps of the generation are as follows:

1. Since the systematic base structure of the generated trace metamodels is known from the domain-specific trace metamodel generator, initialize can be generated;

2. Since the mutable fields of the execution metamodel and the corresponding classes in the trace metamodel are known, addState can be generated. An implementation of this service includes looking for changes among mutable fields then creating a state and new values if any change is detected. Likewise, revertModelToState can be generated, which relies on links from the trace to the model to restore values and re-create objects.

3. Since the operational semantics and the corresponding step classes in the trace metamodel are known, step creation can be generated. While addSmallStep is straightforward, bigStepStarted requires stacking big steps that are in progress, and to unstack them in bigStepEnded.

4. Finally, since the systematic shape of generated trace metamodels is known, a generic trace metamodel interface can be provided, as defined in Section 3.5

\subsection{Generic Multidimensional Omniscient Debugger}

The last component to define is the generic multidimensional omniscient debugger ( $\mathrm{f}$ in Figure 4) that relies on the execution engine to control the current execution, on the state manager to restore previous states, and on the generic trace metamodel interface to manipulate traces.

Table 1 provides a precise definition of each service required for multidimensional omniscient debugging using the services of the three aforementioned required components. These components are represented by three singletons: engine represents the execution engine, trace represents the root element of a model conforming to the generic trace metamodel, and manager represents the state manager. In the following paragraphs, we explain the definitions of all the services provided by the debugger defined in Table 1

Jump services Table 1 starts with the definition of the most important omniscient debugging service, jump. Jumping consists of going back to a chosen state in the execution trace, and is accomplished via the jump ToState service. First, it uses the restoreModelToState service from the state manager to modify the model, then updates the debugger state represented by currentForwardStep and currentBackwardStep. Additionally, we need to be able to jump back either right before or after an execution step, which is provided by the services jumpBeforeStep and jumpAfterStep. 


\begin{tabular}{|c|c|}
\hline Omniscient Debugging Service & Definition \\
\hline jumpToState(state : ExecutionState) & 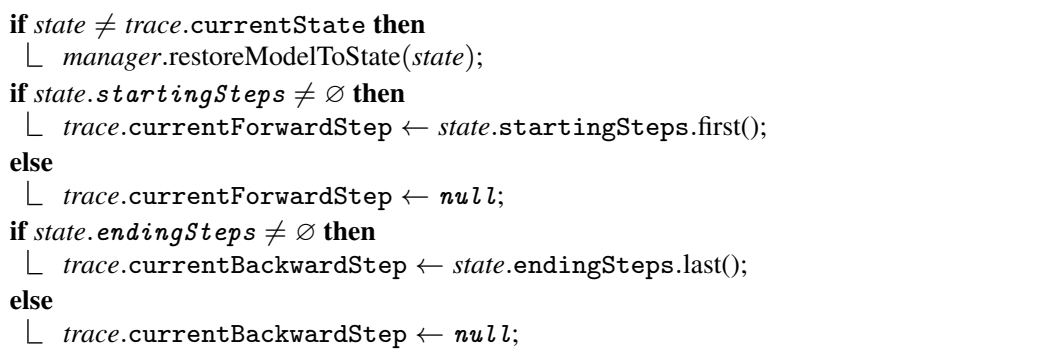 \\
\hline jumpBeforeStep(step : ExecutionStep) & $\begin{array}{l}\text { jumpToState(step.startingState); } \\
\text { trace.currentForwardStep } \leftarrow \text { step; }\end{array}$ \\
\hline jumpAfterStep(step : ExecutionStep) & $\begin{array}{l}\text { if } \text { step.endingState } \neq \text { null then } \\
\qquad \begin{array}{l}\text { jumpToState }(\text { step.endingState }) \\
\text { trace.currentBackwardStep } \leftarrow \text { step }\end{array}\end{array}$ \\
\hline backInto() & jumpAfterStep(trace.currentBackwardStep.previousEnding) \\
\hline backOver() & jumpBeforeStep(trace.currentBackwardStep) \\
\hline backOut () & $\begin{array}{l}\text { if } \text { trace.currentBackwardStep.parent } \neq \text { null then } \\
\qquad \begin{array}{l}\text { trace.currentBackwardStep } \leftarrow \text { trace.currentBackwardStep.parent; } \\
\text { backOver }() ;\end{array}\end{array}$ \\
\hline playBackwards () & 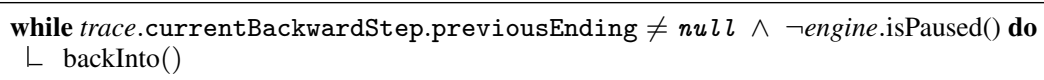 \\
\hline Standard Debugging Service & Definition \\
\hline $\operatorname{toggleBreakpoint}(p:$ Predicate $)$ & engine pauseWhen $(p)$ \\
\hline stepInto() & $\begin{array}{l}\text { if trace.currentForwardStep.nextStarting }=n u \iota l \text { then } \\
\qquad \begin{array}{l}\text { steppedInto } \leftarrow \text { trace.currentForwardStep; } \\
\text { engine.pauseWhen }(\text { steppedInto.nextStarting } \neq \text { null); } \\
\text { engine.resume }() ;\end{array} \\
\text { else } \\
\mathrm{L} \text { jumpBeforeStep (trace.currentForwardStep.nextStarting); }\end{array}$ \\
\hline stepOver() & $\begin{array}{l}\text { if } \text { trace. currentForwardStep.endingState }=\text { null then } \\
\qquad \begin{array}{l}\text { steppedOver } \leftarrow \text { trace.currentForwardStep; } \\
\text { engine.pauseWhen }(\text { steppedOver.endingState } \neq n u l l ; \\
\text { engine.resume }() ;\end{array} \\
\text { else } \\
L \text { jumpAfterStep }(\text { trace.currentForwardStep); }\end{array}$ \\
\hline stepOut() & $\begin{array}{l}\text { if } \text { trace. currentForwardStep.parent } \neq \text { null then } \\
\qquad \begin{array}{l}\text { trace. currentForwardStep } \leftarrow \text { trace. } \text { currentForwardStep.parent; } \\
\text { stepOver }() ;\end{array}\end{array}$ \\
\hline play() & 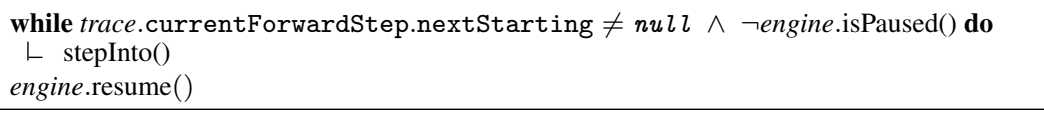 \\
\hline Multidim. Omniscient Debugging Service & Definition \\
\hline jumpToValue $(v:$ Value $)$ & jumpToState $(v$.executionStates.first ()$)$ \\
\hline stepValue(valueSeq : ValueSequence) & $\begin{array}{l}\text { if } \text { valueSeq. current.nextValue }=\text { null then } \\
\qquad \begin{array}{l}\text { previousValue } \leftarrow \text { valueSeq. current; } \\
\text { engine.pauseWhen }(\text { previousValue.nextValue } \neq n u l l ; \\
\text { engine.resume }() ;\end{array} \\
\text { else } \\
L \text { jumpToValue(valueSeq.current.nextValue); }\end{array}$ \\
\hline backValue(valueSeq : ValueSequence) & jumpToValue(valueSeq.current.previousValue) \\
\hline
\end{tabular}

Table 1: Definition of the Generic Multidimensional Omniscient Debugger 


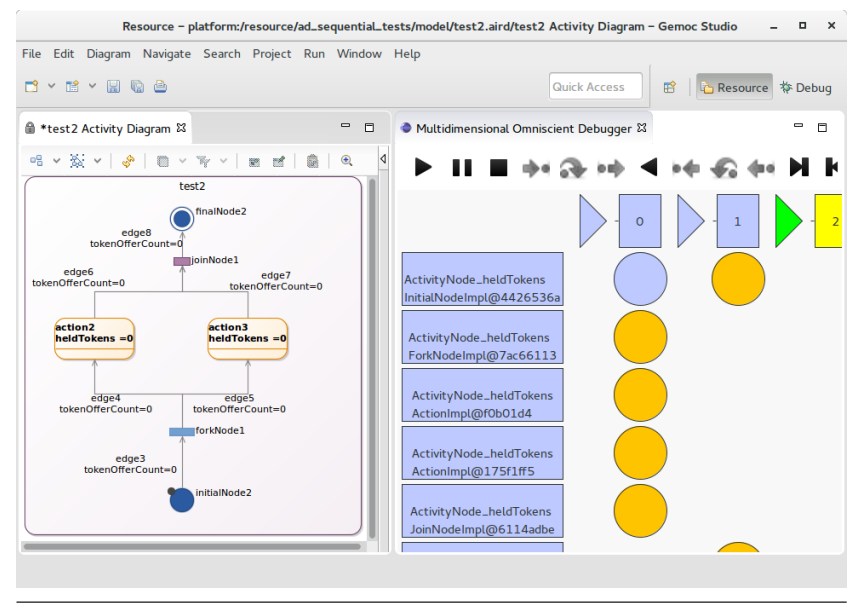

Figure 8. GEMOC Studio with the multidimensional omniscient debugger prototype running an fUML activity.

Other omniscient debugging services Next, we define the remaining omniscient debugging services. backlnto, backOver and backOut directly rely on jumps to reach the correct state. The last service, playBackwards, is a loop backwards until either the initial state is reached or the engine is paused.

Standard debugging services The second tiers of Table 1 define the standard debugging services; i.e., breakpoints and forward stepping. toggleBreakpoint provides a generic way to define a breakpoint through a predicate, that can be defined on the model state (e.g., watching for a specific instruction to be reached) or on the trace (e.g., verifying a temporal property or watching for a specific step to be applied). It is defined using the pauseWhen service that must be provided by the execution engine. The next services are the standard step operations: steplnto, stepOver, and stepOut. There are two cases to consider: (1) When the current step is at the end of the trace, we rely on pauseWhen and resume to apply the operational semantics up until the correct situation is reached (e.g., waiting for the current big step to be finished with stepOver). (2) When the execution state is at a past state (e.g., after a jump backwards), jump services are called (even though these step services are not specific to omniscient debugging) while the engine remains paused.

Multidimensional omniscient debugging services The last tiers of Table 1 define the final set of services providing multidimensional omniscient debugging facilities. The goal of these services is to provide the capacity to debug a model by following the sequences of values of specific mutable fields, thereby improving the usability of omniscient debugging for $x D S M L s$ O\#2. Implementing these services is simplified by the structure of the trace metamodel providing access to each of the value sequences. Thus, jump ToValue is a use of jumpToState; and backValue directly uses jumpToValue; while stepValue is very similar to stepOver.

\section{Tooling for Omniscient Debugging}

This section presents the language and modeling workbench called GEMOC Studio and explains how we applied a subset of our approach (i.e., the generative part and a debugger with basic operations) to offer a proof-of-concept prototype multidimensional omniscient debugger.

\subsection{The GEMOC Studio}

The GEMOC Studi ${ }^{3}$ is an Eclipse package atop the Eclipse Modeling Framework (EMF) including both a language workbench to design and implement tool-supported xDSMLs as well as a modeling workbench where the xDSMLs are automatically deployed to allow system designers to edit, execute, simulate, and animate their models. The modeling workbench includes an advanced generic execution engine that can be used to execute any model conforming to an $\mathrm{xDSML}$ defined within the language workbench. An API is available to extend the engine with addons through the use of an observer pattern: the engine sends notifications to all its addons to inform them about the execution progress (e.g., a step is starting/ending). Lastly, it supports pausing and resuming the execution between steps.

Our prototype focused on the operational semantics implementation language Kermeta [9] that relies on aspects to modularly implement the operational semantics and weave them into the provided metamodel.

\subsection{Omniscient Debugging in the GEMOC Studio}

The prototype demonstrating our approach to support multidimensional omniscient debugging is implemented in GEMOC Studio. The prototype requires the creation and integration of the following components.

Trace addon generator The generative part of our approach takes the form of a trace addon generator, that takes as input an xDSML composed of an Ecore abstract syntax and Kermeta aspects. Note that Kermeta aspects function both as an execution metamodel and operational semantics, because aspect weaving allows the definition of new mutable properties in classes and operations used as transformation rules. The generator produces a GEMOC engine addon with a domain-specific trace metamodel and a domain-specific trace manager. Using the addon mechanism, the manager reacts to the execution steps of the engine to construct the trace accordingly. It also provides an interface to revert the state of the model (i.e., a state manager), and an interface to query the trace (i.e., a generic trace metamodel interface).

Generic debugger logic The generic part of our approach includes multidimensional omniscient debugging services within the GEMOC studio. Our prototype provides toggleBreakpoint with only one kind of predicate (i.e., a model element is targeted by a step), steplnto, jump ToState, jumpToValue, and visualization (see next paragraph).

\footnotetext{
3 http://gemoc.org/studio
} 
Graphical interface The graphical user interface of the debugger includes a prototypical graphical widget that shows both the execution trace and the value sequences of all mutable fields. Figure 8 shows the GEMOC Studio with a model animator on the left and the omniscient debugging widget on the right. Double clicking on a model element triggers a toggleBreakpoint that pauses the execution when this element is targeted by a step. In the right widget showing the trace, the first row of numbered squares represents all the execution states. Each subsequent row represents the value sequence of a specific mutable field. The yellow rectangle indicates the current execution state and orange circles indicate current values of the mutable fields. Double-clicking on a state (numbered squares) triggers a jumpToState to the corresponding execution state. Doing similarly on one of values (circles) triggers a jumpToValue to the corresponding value. Since the GEMOC Studio provides animation of the executed model, the left view showing the model is updated at each action.

Additional information about our prototype can be found on the companion web page of this paper: http://gemoc.org/sle15-omniscientdebugging/

\section{Evaluation}

In this section, we first present the design and results of an empirical study providing an initial evaluation of the efficiency of our approach. Then, we discuss the benefits of multidimensional omniscient debugging.

\subsection{Efficiency of the Approach}

To evaluate the efficiency of our approach O\#1, we considered the following research questions:

RQ\#1: Is our approach more efficient in memory as compared to a clone-based omniscient debugger?

RQ\#2: Is our approach more efficient in time for omniscient debugging services as compared to a weak omniscient debugger and to a clone-based omniscient debugger?

Thus, our evaluation of efficiency is the comparison of three omniscient debuggers as presented in Section 2.2 and in Figure 3. First, WeakDebugger is a weak generic omniscient debugger. Such a debugger is expected to be efficient in memory, because there is no trace to store; and inefficient in time, because the execution engine must be restarted at each jump backward. Second, CloneBasedDebugger is a clone-based generic omniscient debugger, that constructs a generic trace using deep cloning (i.e., the complete model is copied at each step) and implements jumps using the model differencing library EMF Compare 4 , Because this debugger relies on an execution trace, it is expected to be less efficient in memory and more efficient in time than WeakDebugger. Finally, MultiDimDebugger is the prototype multidimensional omniscient debugger applying our approach. All three debuggers were implemented in the GEMOC Studio.

\footnotetext{
4 https://www.eclipse.org/emf/compare/
}

We applied our approach to a subset of a real-world xDSML, namely fUML [15]. The considered subset contains the Activity Diagram portion of the language. In summary, a model conforming to this XDSML is made of an activity, which consists of control nodes and action nodes. Nodes are linked by control flow links, starting with an initial node and ending with a final node. Similarly to a Petri Net, tokens are passed along nodes to drive the execution. In addition, variables can be defined in activities and modified with actions. The xDSML was implemented with GEMOC Studio using Ecore for the abstract syntax and Kermeta for both the execution metamodel and the operational semantics.

As in our previous work [2], we used models taken from the case study of Maoz et al. [12]5] This choice was made to help establish a benchmark, facilitate comparison with future work, and because the models were drawn from industrial sources. The dataset we obtain contains 40 models whose sizes range from 36 to 51 objects. We plan to integrate larger models to the dataset for a future study, but are confident in the current ones to provide initial meaningful comparison.

Data Collection and Analysis To compare efficiency in memory, instead of observing the memory usage of the complete environment (e.g., execution engine and loaded model), we measured the memory used only by the debugger. More precisely, for each of the considered models, we collected the amount of memory required to store the execution trace at the end of its execution by making precise memory measurements using heap dumps and Eclipse MAT 6

To compare efficiency in time, we focused on the main operation used by all omniscient debugging services: jumpToState. More precisely, for each of the considered models, we measured the average amount of time required to perform a jump ToState by jumping to each previously visited state once and in a random order. Measures were done using Java's operation System.nanoTime.

Data was collected in a reproducible way through a programmatic use of GEMOC Studio's engine (see the companion webpage). Each result is an average value computed from five identical measurements made using an Intel i7-3720QM CPU with 8GB of RAM.

RQ\#1, Efficiency in memory Figure 9 shows the results obtained regarding the memory required to store an execution trace. The $x$-axis shows the number of elements in the trace, while the $y$-axis shows the amount of memory used in $\mathrm{kB}$. First, WeakDebugger does not use memory, because it does not store a trace. Second, we observe that our approach is always more efficient in terms of memory usage than the CloneBasedDebugger debugger with 3.0 times improvement on average. We hypothesize this is due to the domain-specific traces obtained with our approach that are designed to only contain the evolution of the mutable fields of the model with

\footnotetext{
${ }^{5}$ Models available at http://www.se-rwth.de/materials/semdiff/ 6 https : //www.eclipse.org/mat/
} 


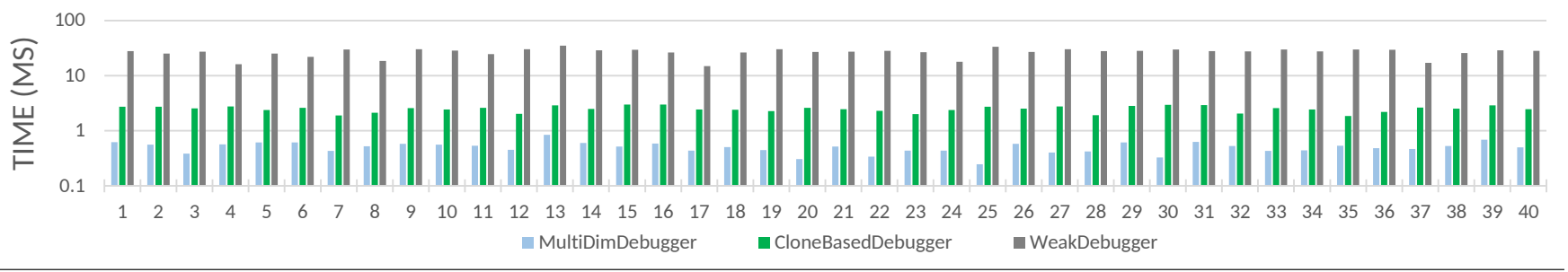

Figure 10. Time required to perform a jumpToState

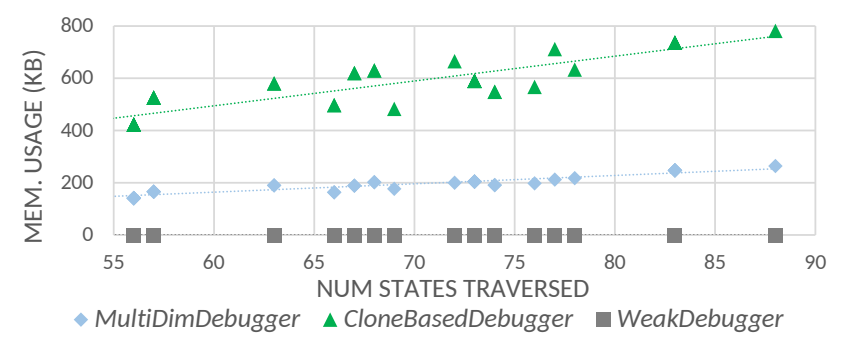

Figure 9. Memory used by the execution trace

minimal redundancy, whereas cloning implies significant redundancy. In addition, we note that our approach has a gentler slope than CloneBasedDebugger, which suggests better scalability with large traces. To summarize and answer RQ \#1, we observe that our approach is more efficient in memory than a clone-based approach.

$R Q \# 2$ Efficiency in time Figure 10 presents the results obtained regarding the average amount of time required to perform a jumpToState. The $x$-axis shows the identifier of the executed model, while the $y$-axis shows the amount of time in $m s$. First, we observe that trace-based debuggers are always better than WeakDebugger (right), with in particular MultiDimDebugger (left) being 54.1 times faster than WeakDebugger. This is explained by the time required to reset the execution engine. Second, we observe that MultiDimDebugger is more efficient than CloneBasedDebugger (center) with 5.03 times improvement on average. We hypothesize this is due to the generated trace manager, which contains code specific and tuned to both the XDSML and the generated domain-specific trace metamodel. To summarize and answer RQ \#2, we observe our approach is more efficient in time than the traceless approach and clone-based approach.

\subsection{Benefits of Multidimensional Facilities}

To ensure the usability of omniscient debugging (O\#2), our approach provides multidimensional omniscient debugging; i.e., facilities to navigate among values of mutable fields of an executed model. In essence, we believe that providing explicit visualization of the dimensions of a trace (see Figure 8) and means to traverse such trace according to specific dimensions (e.g., stepValue), has a significant positive impact on usability $\mathrm{O \# 2}$. To completely validate $\mathrm{O \# 2}$ requires user experiments to empirically assess the expected benefits of multidimensional facilities. We defer this task to future work.

\section{Related Work}

In this section, we overview the existing work in the MDE literature on debugging and on execution trace management.

\subsection{Omniscient Debugging in MDE}

Maoz and Harel [11] and Hegedüs et al. [7] present trace exploration tools which contain similar facilities to an omniscient debugger. However, these techniques are defined for post-mortem analysis rather than use during live sessions, whereas our technique supports live debugging sessions. Additionally, Maoz and Harel do not support domain-specific execution traces for xDSMLs.

In our previous work [6], we explored applying omniscient debugging to model transformations within the context of AToMPM, a multi-paradigm modeling tool. Yet the focus was the two basic transformation languages provided by AtoMPM, while in this paper we are concerned with XDSMLs. We are not aware of any other literature that applied omniscient debugging in the context of MDE.

\subsection{Trace Visualization and Debugging in MDE}

Existing work on trace visualization, such as MetaViz by Aboussoror et al. [1], or the work of Maoz and Harel [11], would be strongly complimentary with our approach. Indeed, while we focused on the backend concern of omniscient debugging, trace visualization is required for the frontend.

More recently, the work of Chis et al. on a Moldable Debugger [3] can be interestingly compared to our work. Indeed, while we provide generic debugging operations supported by domain-specific trace management facilities, they provide a framework to define domain-specific debugging operations and user interfaces. Also, our approach is completely automatic given a well-formed xDSML, whereas manual work is required to extend the Moldable Debugger to support an xDSML. Yet, both approaches tackle different and independent challenges, and provide very complementary results.

\subsection{Domain-Specific Execution Traces in MDE}

Meyers et al. introduced the ProMoBox framework [14], which generates a set of metamodels from an annotated xDSML, including domain-specific trace metamodel. Among 
others, a difference with our work is that they consider an annotated abstract syntax whose properties are annotated either as runtime or event, while we consider the abstract syntax and the execution metamodel to be separated for better separation of concerns. In addition, they do not provide alternative ways to explore a trace, while we provide various navigation paths for multidimensional debugging.

Similarly, Gogolla et al. [8] generate filmstrip models from UML class diagrams. Such filmstrip models match what we call domain-specific trace metamodels, and provide some navigation paths among objects states. However, they do not tackle redundancy since object states are always recreated at each model change, and they do not consider storing only values of mutable fields.

\section{Conclusion and Future Work}

Omniscient debugging is a promising dynamic $\mathrm{V} \& \mathrm{~V}$ approach for xDSMLS that enables free traversal of the execution of a system. While most GPLs already have efficient debuggers, bringing omnicient debugging to any XDSML is a tedious and error-prone task. A solution is to define a purely generic debugger, but this requires managing both efficiency and usability issues that emerge. The approach we presented relies on generated domain-specific trace management facilities for improved efficiency and provides multidimensional omniscient debugging facilities for improved usability. The debugger relies on an execution engine to control the execution and a generated domain-specific trace manager to provide omniscient services. The states reached during an execution are stored in a trace conforming to a generated domain-specific trace metamodel. We provide a prototype within GEMOC Studio, a language and modeling workbench, and an evaluation performed using the fUML language. We observed an improvement regarding both the memory consumption and the time to perform a jump, when compared to two generic omniscient debugger variants.

The direct perspectives of this work include taking advantage of the underlying domain-specific trace metamodel to supplement the debugger with domain-specific tooling, user experiments to empirically assess the expected benefits of multidimensional facilities, and adapting omniscient debugging to support both external stimuli and the concurrency model in operational semantics [5]; e.g., to explore the possible executions traces of a single executable model.

\section{Acknowledgment}

This work is partially supported by the ANR INS Project GEMOC (ANR-12-INSE-0011).

\section{References}

[1] E. Aboussoror, I. Ober, and I. Ober. Seeing Errors: Model Driven Simulation Trace Visualization. In 15th Int. Conf. on Model Driven Eng. Lang. and Sys. (MODELS), volume 7590 of LNCS. Springer, 2012.
[2] E. Bousse, T. Mayerhofer, B. Combemale, and B. Baudry. A Generative Approach to Define Rich Domain-Specific Trace Metamodels. In 11th Eur. Conf. on Modelling Foundations and Applications (ECMFA), volume 9153 of LNCS. Springer, 2015.

[3] A. Chis, T. Gîrba, and O. Nierstrasz. The Moldable Debugger: a Framework for Developing Domain-Specific Debuggers. In 7th Int. Conf. on Soft. Lang. Eng. (SLE), volume 8706 of LNCS. Springer, 2014.

[4] B. Combemale, X. Crégut, and M. Pantel. A Design Pattern to Build Executable DSMLs and Associated V\&V Tools. In 19th Asia-Pacific Soft. Eng. Conf. (APSEC), volume 1. IEEE, 2012.

[5] B. Combemale, J. Deantoni, M. V. Larsen, F. Mallet, O. Barais, B. Baudry, and R. France. Reifying Concurrency for Executable Metamodeling. In 6th Int. Conf. on Soft. Lang. Eng. (SLE), volume 8225 of $L N C S$. Springer, 2013.

[6] J. Corley, B. Eddy, and J. Gray. Exploring Omniscient Debugging for Model Transformations. In 14th Workshop on Domain-Specific Modeling, volume 1258. CEUR, 2014.

[7] Á. Hegedüs, I. Ráth, and D. Varró. Replaying Execution Trace Models for Dynamic Modeling Languages. Periodica Polytechnica-Electrical Eng., 56, 2012.

[8] F. Hilken, L. Hamann, and M. Gogolla. Transformation of UML and OCL Models into Filmstrip Models. In 7th Int. C. on Model Transf. (ICMT), volume 8568 of LNCS. Springer, 2014.

[9] J.-M. Jézéquel, B. Combemale, O. Barais, M. Monperrus, and F. Fouquet. Mashup of metalanguages and its implementation in the Kermeta language workbench. Soft. \& Sys. Modeling (SoSyM), 14(2), 2013.

[10] A. Lienhard, T. Gîrba, and O. Nierstrasz. Practical ObjectOriented Back-in-Time Debugging. In 22nd Eur. Conf. on Object-Oriented Programming (ECOOP), volume 5142 of LNCS. Springer, 2008.

[11] S. Maoz and D. Harel. On tracing reactive systems. Soft. \& Sys. Modeling (SoSyM), 10(4), 2011.

[12] S. Maoz, J. O. Ringert, and B. Rumpe. ADDiff: Semantic Differencing for Activity Diagrams. In 19th ACM SIGSOFT Symposium and the 13th Eur. Conf. on Foundations of Soft. Eng. (ESEC/FSE). ACM, 2011.

[13] T. Mayerhofer, P. Langer, M. Wimmer, and G. Kappel. xMOF: Executable DSMLs based on fUML. In 6th Int. Conf. on Soft. Lang. Eng. (SLE), volume 8225 of LNCS. Springer, 2013.

[14] B. Meyers, R. Deshayes, L. Lucio, E. Syriani, H. Vangheluwe, and M. Wimmer. ProMoBox: A Framework for Generating Domain-Specific Property Languages. In 7th Int. Conf. on Soft. Lang. Eng. (SLE), volume 8706 of LNCS. Springer, 2014.

[15] Object Management Group. Semantics of a Foundational Subset for Executable UML Models, V 1.1, August 2013.

[16] G. Pothier and E. Tanter. Back to the Future: Omniscient Debugging. IEEE Soft., 26(6), nov 2009.

[17] J. Tatibouët, A. Cuccuru, S. Gérard, and F. Terrier. Formalizing Execution Semantics of UML Profiles with fUML Models. In 17th Int. Conf. on Model Driven Eng. Lang. and Sys. (MODELS), volume 8767 of LNCS. Springer, 2014. 